\title{
DYNAMIC CRACK PROPAGATION \\ IN SHEETS OF COMPRESSIBLE NEO-HOOKEAN MATERIAL UNDER GENERAL IN-PLANE LOADING
}

BY

\author{
ANGELO MARCELLO TARANTINO
}

Dipartimento di Scienze dell'Ingegneria, Università di Modena e Reggio Emilia, Modena, Italy

1. Introduction. From the few in-plane crack problems solved in finite elastostatics a substantial difference emerges with respect to the predictions of the classic linear theory of elasticity: the nonexistence of antisymmetric asymptotic solutions. In other words, due to the nonlinearities, the crack-face opening, near the crack-tip, is to be expected even when the applied loading is antisymmetric about the crack line.

In 1982, Stephenson [1] succeeded in proving this important result for a class of incompressible Mooney-Rivlin materials under the plane strain condition. Successively, the same result was obtained, again under the plane strain condition, by Le for a Hadamard material [2], by Le and Stumpf for a class of Ogden-Ball rubberlike materials [3], and, under the plane stress condition, by Geubelle and Knauss for a generalized neo-Hookean incompressible material $[4,5,6]$ and by Tarantino for a compressible neo-Hookean material [7].

All the nonlinear crack analyses cited gave the somewhat surprising result that the near-tip deformation field $\mathbf{f}$ is obtained through a mere rigid transformation of the canonical symmetric asymptotic field $\mathbf{f}^{*}$. At the crack-tip the deformation field then assumes the following asymptotic representation form:

$$
\mathbf{f}=\mathbf{f}^{0}+\mathbf{Q \mathbf { f } ^ { * }}
$$

where the rigid translation $\mathbf{f}^{0}$ and the rigid rotation $\mathbf{Q}$ depend on the far-field loading conditions (see Fig. 1). If only the dominant order terms are taken into account, the canonical field $\mathbf{f}^{*}$ represents the particular deformation field in which the crack faces open symmetrically, satisfying the symmetry properties

$$
f_{1}^{*}(r, \theta)=f_{1}^{*}(r,-\theta) \text { and } f_{2}^{*}(r, \theta)=-f_{2}^{*}(r,-\theta),
$$

just like Mode I in linear elastic fracture mechanics. It is important to note that (1.1) holds regardless of the type of assigned loading conditions (that is, for Mode I loading, Mode II loading, or any combination).

Received July 29, 1999.

2000 Mathematics Subject Classification. Primary 74B20, 74G70, 74R15. 


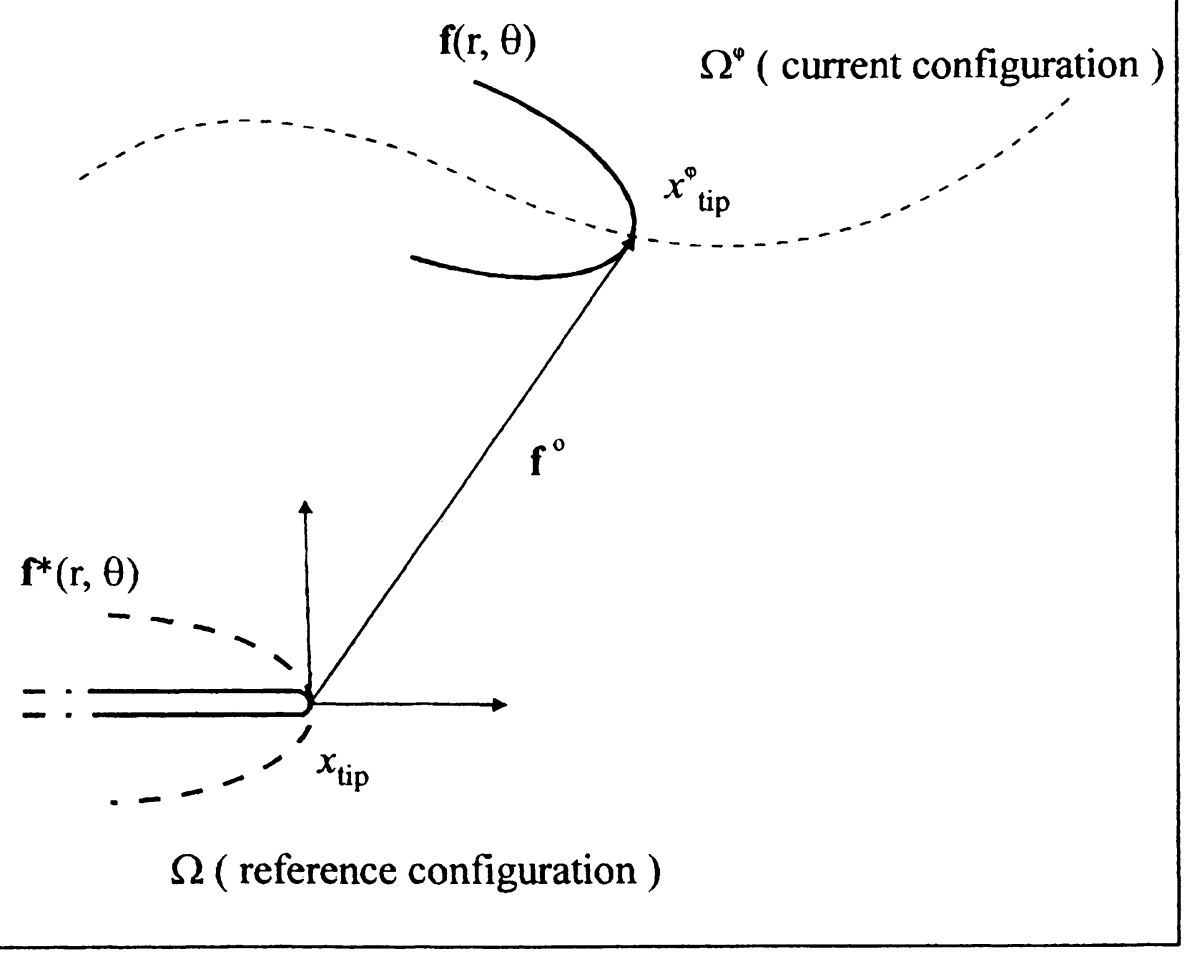

FIci. 1. Graphic representation of the relation $\mathbf{f}=\mathbf{f}^{0}+\mathbf{Q} \mathbf{f}^{*}$

Stephenson's result shows how the peculiarity of the nonlinear behaviour, for a wide class of cracked bodies, results mainly in the impossibility of the antisymmetric mode (or Mode II), so that mixed-mode interaction leading to crack kinking is also impossible. Given the symmetries of the canonical field and of the material properties, and excluding the branching, a propagating crack-tip, in the reference configuration, will necessarily move following the straight path, and this for every type of loading condition.

This particular implication motivates the present work, in which a crack propagation analysis in the context of finite elastodynamics is performed. More specifically, a straight crack, that suddenly grows at constant velocity in a thin sheet of compressible neoHookean material, is studied. A similar plane crack problem so far does not seem to have been treated; while, a steady-state dynamic crack propagation problem, for an elasticplastic material under the antiplane deformation condition (Mode III), was investigated by Achenbach and Nishimura [8, 9].

This paper is organized as follows. The local formulation of the two-dimensional boundary-initial-value problem is stated in Sec. 2, where the growing crack in the reference configuration is modelled by a rectilinear increasing line of discontinuity. ${ }^{1}$ The

\footnotetext{
${ }^{1}$ Consequently, for this class of problems even the reference configuration is time-dependent.
} 
equation of motion, the boundary and initial conditions are expressed with respect to a coordinate system which translates with such a line and whose origin is attached to the apex. In Sec. 3, an asymptotic analysis is carried out by pursuing the local motion field around the moving crack-tip. The dynamic version of Stephenson's result and the related canonical motion field are discussed in Sec. 4, where the expressions that describe the asymptotic crack profile during propagation are also obtained. Finally, in Sec. 5, the asymptotic Piola-Kirchhoff and Cauchy stress fields around the moving crack-tip, together with the related orders of stress singularity and the vector of energy flux, are computed.

2. Local formulation of the nonlinear crack propagation problem. As already mentioned, our attention will be confined to thin hyperelastic sheets, under the plane stress condition, composed of homogeneous, isotropic material, whose stored energy function $\mathcal{W}$ has the compressible neo-Hookean form [10]

$$
\mathcal{W}(\mathbf{F})=a\|\mathbf{F}\|^{2}+\Gamma(\operatorname{det} \mathbf{F}) \quad \text { for all } \mathbf{F} \in \operatorname{Lin}^{+},{ }^{2}
$$

with

$$
\|\mathbf{F}\|^{2}=\lambda_{1}^{2}+\lambda_{2}^{2}+\lambda_{3}^{2}, \quad \operatorname{det} \mathbf{F}=\lambda_{1} \lambda_{2} \lambda_{3}=\delta,
$$

where $\lambda_{k}$ are the principal stretches of the deformation gradient $\mathbf{F}$. In (2.1), a denotes a strictly positive constant and $\Gamma:] 0, \infty[\rightarrow \mathbb{R}$ is a convex function, for which we assume the expression proposed in [11]

$$
\Gamma(\delta)=c \delta^{2}-d \log \delta
$$

where $c$ and $d$ are another two strictly positive constants.

From (2.1) the following response function for the Piola-Kirchhoff stress tensor $\mathbf{T}_{\mathbb{R}}$ is derived:

$$
\mathbf{T}_{\mathbb{R}}(\mathbf{F})=2 a \mathbf{F}+\Gamma_{, \delta}(\delta) \delta \mathbf{F}^{-\mathrm{T}}
$$

where $\Gamma_{, \delta}=d \Gamma / d \delta$. The mechanical behaviour of the material described by (2.3) is of a hardening-type, as can be observed by imposing a three-dimensional homogeneous deformation corresponding to a uni-axial stress and evaluating the stress response in terms of Cauchy stresses (cf. [12] p. 269). The differences with respect to a classic incompressible neo-Hookean material are illustrated in [7].

To maintain a state of plane stress, the components of the deformation gradient $\mathbf{F}$ are found to be $[13,14]^{3}$

$$
F_{\alpha \beta}=\varphi_{\alpha, \beta}, \quad F_{\alpha 3}=F_{3 \gamma}=\mathbf{0}, \quad F_{33}=\varphi_{3,3}=\lambda\left(x_{1}, x_{2}, t\right) .4
$$

The last quantity denotes the principal stretch with the $x_{3}$-axis as the associated principal axis. To compute $\lambda$ we can use the following plane invariants:

$$
h=\operatorname{tr}\left(B_{\alpha \beta}\right)=\lambda_{1}^{2}+\lambda_{2}^{2}, \quad i=\operatorname{det}\left(F_{\alpha \beta}\right)=\lambda_{1} \lambda_{2},
$$

\footnotetext{
${ }^{2} \mathrm{Lin}^{+}$is the multiplicative group of (second-order) tensors, that is, all linear transformations from the vector space $\mathcal{V}$ into $\mathcal{V}$, with positive determinant.

${ }^{3}$ These components must be considered as their restriction to the middle plane. A modern and accurate description of the fundamental relations of nonlinear plane stress theory can be found in a paper by Knowles and Sternberg [12].

${ }^{4}$ The range of the Greek indices is $\{1,2\}$.
} 
and successively impose the approximate assumption $T_{\mathbb{R}_{33}}=0$, dictated by the plane stress condition, obtaining

$$
\lambda=\left(\frac{d}{2\left(a+c i^{2}\right)}\right)^{\frac{1}{2}} .
$$

In view of (2.3) and (2.4), the non-vanishing components of the Piola-Kirchhoff stress tensor are $\left(T_{\mathbb{R}_{\alpha 3}}=T_{\mathbb{R}_{3 \beta}}=T_{\mathbb{R}_{33}}\right)=0$

$$
T_{\mathbb{R}_{\alpha \beta}}=2 a F_{\alpha \beta}+\left[2 i c \lambda^{2}-\frac{d}{i}\right] \varepsilon_{\alpha \mu} \varepsilon_{\beta \nu} F_{\mu \nu}
$$

since $F_{\alpha \beta}^{-\mathrm{T}}=i^{-1}(\operatorname{Cof} F)_{\alpha \beta}=i^{-1} \varepsilon_{\alpha \mu} \varepsilon_{\beta \nu} F_{\mu \nu}$ (the symbol $\varepsilon_{\alpha \mu}$ is the two-dimensional alternator, i.e., $\varepsilon_{11}=\varepsilon_{22}=0, \varepsilon_{12}=-\varepsilon_{21}=1$ ). By substituting (2.6) into the equation of balance of linear momentum, in the absence of body forces,

$$
\operatorname{Div} \mathbf{T}_{\mathbb{R}}=\rho_{0} \frac{\partial^{2} \boldsymbol{\varphi}}{\partial t^{2}}
$$

where $\rho_{0}$ is the referential mass density, the following equations in terms of components of the motion $\varphi_{\alpha}\left(x_{1}, x_{2}, t\right)$ are derived: ${ }^{5}$

$$
\begin{aligned}
& 2 a \Delta \varphi_{1}=-\psi_{, 1} \varphi_{2,2}+\psi_{, 2} \varphi_{2,1}+\rho_{0} \varphi_{1, t t}, \\
& 2 a \Delta \varphi_{2}=\psi_{, 1} \varphi_{1,2}-\psi_{, 2} \varphi_{1,1}+\rho_{0} \varphi_{2, t t},
\end{aligned}
$$

where

$$
h=\varphi_{1,1}^{2}+\varphi_{1,2}^{2}+\varphi_{2,1}^{2}+\varphi_{2,2}^{2}, \quad i=\varphi_{1,1} \varphi_{2,2}-\varphi_{1,2} \varphi_{2,1}, \quad \psi=2 c i \lambda^{2}-\frac{d}{i},
$$

and $\Delta$ denotes the two-dimensional Laplace operator. The elastodynamic plane stress problem for a compressible neo-Hookean material is governed by the above quasilinear system of coupled partial differential equations.

In this paper, as mentioned in the foregoing section, we exploit Stephenson's result to formulate a dynamic crack propagation problem for a straight crack that suddenly begins to grow, following a collinear direction in the reference configuration, at constant crack speed $v$. Since the principal interest of the local analysis of a crack propagation problem is to study how the motion field around the moving tip evolves in time, it is necessary to express the equations of motion (2.7) with respect to the moving coordinate system indicated in Fig. 2.

Upon adoption of the Galilean variables $x_{1}^{\prime}=x_{1}-v t, x_{2}^{\prime}=x_{2}$ and $t^{\prime}=t$ in the transformation $\varphi_{\alpha}\left(x_{1}, x_{2}, t\right) \rightarrow \hat{\varphi}_{\alpha}\left(x_{1}^{\prime}, x_{2}^{\prime}, t^{\prime}\right)$, equations (2.7) become

$$
\begin{aligned}
& \left(2 a-\rho_{0} v^{2}\right) \frac{\partial^{2} \hat{\varphi}_{1}}{\partial x_{1}^{\prime 2}}+2 a \frac{\partial^{2} \hat{\varphi}_{1}}{\partial x_{2}^{\prime 2}}=-\frac{\partial \hat{\psi}}{\partial x_{1}^{\prime}} \frac{\partial \hat{\varphi}_{2}}{\partial x_{2}^{\prime}}+\frac{\partial \hat{\psi}}{\partial x_{2}^{\prime}} \frac{\partial \hat{\varphi}_{2}}{\partial x_{1}^{\prime}}-2 \rho_{0} v \frac{\partial^{2} \hat{\varphi}_{1}}{\partial x_{1}^{\prime} \partial t^{\prime}}+\rho_{0} \frac{\partial^{2} \hat{\varphi}_{1}}{\partial t^{\prime 2}}, \\
& \left(2 a-\rho_{0} v^{2}\right) \frac{\partial^{2} \hat{\varphi}_{2}}{\partial x_{1}^{\prime 2}}+2 a \frac{\partial^{2} \hat{\varphi}_{2}}{\partial x_{2}^{\prime 2}}=\frac{\partial \hat{\psi}}{\partial x_{1}^{\prime}} \frac{\partial \hat{\varphi}_{1}}{\partial x_{2}^{\prime}}-\frac{\partial \hat{\psi}}{\partial x_{2}^{\prime}} \frac{\partial \hat{\varphi}_{1}}{\partial x_{1}^{\prime}}-2 \rho_{0} v \frac{\partial^{2} \hat{\varphi}_{2}}{\partial x_{1}^{\prime} \partial t^{\prime}}+\rho_{0} \frac{\partial^{2} \hat{\varphi}_{2}}{\partial t^{\prime 2}} .
\end{aligned}
$$

By virtue of such a transformation the boundary conditions on the crack surfaces assume a simpler form. Moreover, as usual in fracture mechanics, polar coordinates are

${ }^{5}$ Differentiation with respect to a variable $x_{i}$ is shown by an index $i$ preceded by a comma. 


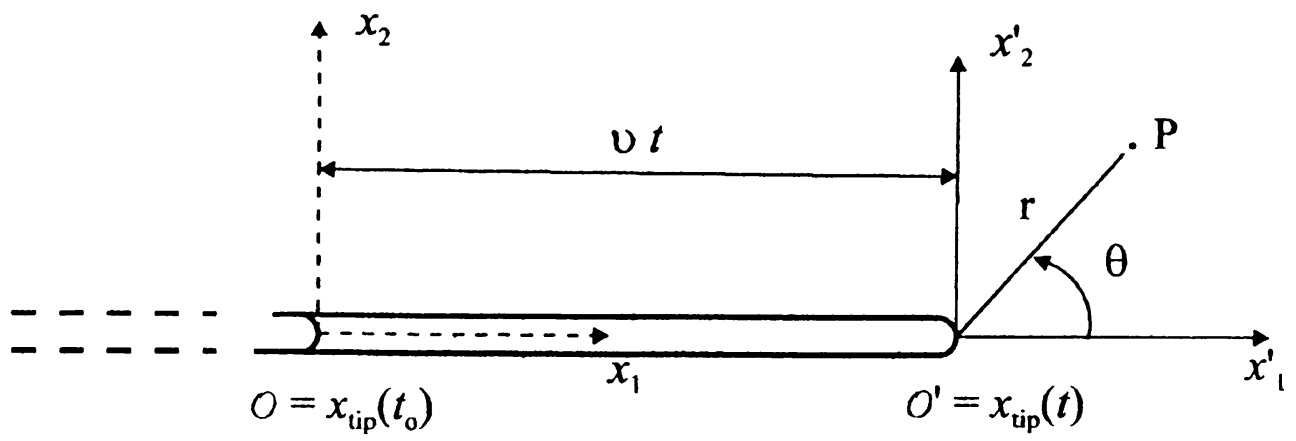

FIG. 2. Rectilinear crack propagation through the reference configuration

adopted to express the motion, that is, $\hat{\varphi}_{\alpha}\left(x_{1}^{\prime}, x_{2}^{\prime}, t^{\prime}\right) \rightarrow \hat{\hat{\varphi}}_{\alpha}\left(r, \theta, t^{\prime \prime}\right)$. (In the sequel, the superscripts of $\hat{\hat{\varphi}}_{\alpha}$ and of $t^{\prime \prime}$ will be omitted.) Thus, equations (2.9) are rewritten as

$$
\begin{gathered}
2 a\left[\varphi_{1, r r}+\frac{1}{r} \varphi_{1, r}+\frac{1}{r^{2}} \varphi_{1, \theta \theta}\right]=-\frac{1}{r} \psi_{, r} \varphi_{2, \theta}+\frac{1}{r} \psi_{, \theta} \varphi_{2, r} \\
+\rho_{0}\left[v^{2}\left(\varphi_{1, r r} \cos ^{2} \theta-\frac{2 \sin \theta \cos \theta}{r} \varphi_{1, \theta r}+\frac{\sin ^{2} \theta}{r^{2}} \varphi_{1, \theta \theta}+\frac{2 \sin \theta \cos \theta}{r^{2}} \varphi_{1, \theta}+\frac{\sin ^{2} \theta}{r} \varphi_{1, r}\right)\right. \\
\left.\quad-2 v\left(\varphi_{1, r t} \cos \theta-\frac{\sin \theta}{r} \varphi_{1, \theta t}\right)+\varphi_{1, t t}\right] \\
2 a\left[\varphi_{2, r r}+\frac{1}{r} \varphi_{2, r}+\frac{1}{r^{2}} \varphi_{2, \theta \theta}\right]=\frac{1}{r} \psi_{, r} \varphi_{1, \theta}-\frac{1}{r} \psi_{, \theta} \varphi_{1, r} \\
+\rho_{0}\left[v^{2}\left(\varphi_{2, r r} \cos ^{2} \theta-\frac{2 \sin \theta \cos \theta}{r} \varphi_{2, \theta r}+\frac{\sin ^{2} \theta}{r^{2}} \varphi_{2, \theta \theta}+\frac{2 \sin \theta \cos \theta}{r^{2}} \varphi_{2, \theta}+\frac{\sin ^{2} \theta}{r} \varphi_{2, r}\right)\right. \\
\left.-2 v\left(\varphi_{2, r t} \cos \theta-\frac{\sin \theta}{r} \varphi_{2, \theta t}\right)+\varphi_{2, t t}\right]
\end{gathered}
$$

Likewise, the plane invariants transform into

$$
h=\left(\varphi_{1, r}^{2}+\varphi_{2, r}^{2}\right)+\frac{1}{r^{2}}\left(\varphi_{1, \theta}^{2}+\varphi_{2, \theta}^{2}\right), \quad i=\frac{1}{r}\left(\varphi_{1, r} \varphi_{2, \theta}-\varphi_{2, r} \varphi_{1, \theta}\right),
$$

and the cartesian components of the Piola-Kirchhoff stress tensor (2.6) become

$$
\begin{aligned}
& T_{\mathbb{R}_{11}}=2 a \varphi_{1, r} \cos \theta-2 a \frac{\sin \theta}{r} \varphi_{1, \theta}+\psi \varphi_{2, r} \sin \theta+\psi \frac{\cos \theta}{r} \varphi_{2, \theta}, \\
& T_{\mathbb{R}_{12}}=2 a \varphi_{1, r} \sin \theta+2 a \frac{\cos \theta}{r} \varphi_{1, \theta}-\psi \varphi_{2, r} \cos \theta+\psi \frac{\sin \theta}{r} \varphi_{2, \theta}, \\
& T_{\mathbb{R}_{21}}=2 a \varphi_{2, r} \cos \theta-2 a \frac{\sin \theta}{r} \varphi_{2, \theta}-\psi \varphi_{1, r} \sin \theta-\psi \frac{\cos \theta}{r} \varphi_{1, \theta}, \\
& T_{\mathbb{R}_{22}}=2 a \varphi_{2, r} \sin \theta+2 a \frac{\cos \theta}{r} \varphi_{2, \theta}+\psi \varphi_{1, r} \cos \theta-\psi \frac{\sin \theta}{r} \varphi_{1, \theta},
\end{aligned}
$$


From (2.12) we can derive the related polar components

$$
\begin{aligned}
& T_{\mathbb{R}_{r, r}}=2 a\left(\varphi_{1, r} \cos \theta+\varphi_{2, r} \sin \theta\right)+\frac{\psi}{r}\left(\varphi_{2, \theta} \cos \theta-\varphi_{1, \theta} \sin \theta\right), \\
& T_{\mathbb{R}_{\theta r}}=2 a\left(-\varphi_{1, r} \sin \theta+\varphi_{2, r} \cos \theta\right)+\frac{\psi}{r}\left(-\varphi_{2, \theta} \sin \theta-\varphi_{1, \theta} \cos \theta\right), \\
& T_{\mathbb{R}_{r \theta}}=\frac{2 a}{r}\left(\varphi_{1 . \theta} \cos \theta+\varphi_{2, \theta} \sin \theta\right)+\psi\left(-\varphi_{2, r} \cos \theta+\varphi_{1, r} \sin \theta\right), \\
& T_{\mathbb{R}_{\theta \theta}}=\frac{2 a}{r}\left(\varphi_{2, \theta} \cos \theta-\varphi_{1, \theta} \sin \theta\right)+\psi\left(\varphi_{1, r} \cos \theta+\varphi_{2, r} \sin \theta\right) .
\end{aligned}
$$

The local crack propagation problem may now be stated by requiring that the motion $\varphi(r, \theta, t)$ satisfies the field equations $(2.10)$, the initial conditions

$$
\varphi\left(r, \theta, t_{0}\right)=\varphi_{0}(r, \theta), \quad \frac{\partial \varphi}{\partial t}\left(r, \theta, t_{0}\right)=\dot{\varphi}_{0}(r, \theta),
$$

where $\varphi_{0}$ and $\dot{\varphi}_{0}$ are prescribed vector-valued functions, and that, at least in proximity of the crack-tip, the surfaces of the crack must be traction-free, namely

$$
\left.T_{\mathbb{R}_{r \cdot \theta}}(r, \theta, t)\right|_{\theta= \pm \pi}=0,\left.\quad T_{\mathbb{R}_{\theta \theta}}(r, \theta, t)\right|_{\theta= \pm \pi}=0,
$$

or, using (2.13),

$$
\left.\left(\frac{\omega}{r} \varphi_{1, \theta}-\psi \varphi_{2, r}\right)\right|_{\theta= \pm \pi}=0,\left.\quad\left(\frac{\omega}{r} \varphi_{2, \theta}+\psi \varphi_{1, r}\right)\right|_{\theta= \pm \pi}=0 .
$$

3. Asymptotic computations. An asymptotic (or local) analysis gives solutions which hold exclusively for points close to the crack-tip. To investigate the asymptotic singularities of the motion field induced by a propagating crack we take the existence of a solution to the dynamic global problem for granted and assume that such a solution, near the moving tip, has the following representation form:

$$
\varphi_{\alpha}(r, \theta, t)=\varphi_{\alpha}^{0}(t)+r^{m} v_{\alpha}(\theta ; v) f_{\alpha}(t ; v)+o\left(r^{m}\right)^{6} \quad \text { as } r \rightarrow 0,
$$

where $\varphi_{\alpha}^{0}$ are the components of an unknown twice continuously differentiable vectorvalued function that indicates the position occupied by the moving tip in the current configuration, and $m$ is an unknown real-valued constant obeying the inequality $0<$ $m<1$. The exponent $m$ is positive. It must in fact not be less than zero or the motion would become infinite as $r \rightarrow 0$, while the assumption $m<1$ ensures that the deformation gradient does not remain bounded as $r \rightarrow 0$. The most important singularity of the deformation gradient corresponds to the smallest exponent $m \in(0,1)$ and, through (2.6), of the Piola-Kirchhoff stress tensor. $\hat{v}_{\alpha}(\theta ; v)$ and $f_{\alpha}(t ; v)$ are twice continuously differentiable real-valued unknown functions that fail to vanish identically on $[-\pi, \pi]$ and on $\left[t_{0}, t_{f}\right)$, respectively. The functions $v_{\alpha}(\theta ; v)$ describe the angular variation of the asymptotic motion field, whereas the functions $f_{\alpha}(t ; v)$ show the variation in time. In both functions, the crack speed $v$ plays the role of an assigned parameter.

We begin asymptotic computations by demanding the validity of asymptotic equalities resulting from partial differentiations of (3.1). Subsequently, by inserting (3.1) into

${ }^{6} O(\cdot)$ and $O(\cdot)$ denote the Landau order symbols. 
$(2.11)_{2}$ and $(2.5)$, and into $(2.8)_{3}$ in order to calculate the derivatives of $\psi$ with respect to the polar variables (that appear in the field equations $(2.10)$ ), one obtains

$$
\begin{gathered}
i(r, \theta, t)=M(\theta, t) r^{2(m-1)}+o\left(r^{2(m-1)}\right), \\
\lambda^{2}(r, \theta, t)=\frac{d}{2\left(a+c M^{2}(\theta, t) r^{4(m-1)}\right)}+o\left(r^{4(1-m)}\right),
\end{gathered}
$$

with $M(\theta, t)=m f_{1}(t ; v) f_{2}(t ; v)\left[v_{1}(\theta ; v) v_{2, \theta}(\theta ; v)-v_{1, \theta}(\theta ; v) v_{2}(\theta ; v)\right]$, and

$$
\begin{gathered}
r \psi_{, r}=\left\{\frac{2 c d(m-1) M}{\left(a r^{4(1-m)}+c M^{2}\right)}-\frac{4 c^{2} d(m-1) M^{3}}{\left(a r^{4(1-m)}+c M^{2}\right)^{2}}+\frac{2 d(m-1)}{M}\right\} r^{2(1-m)}+o\left(r^{2(1-m)}\right), \\
\psi_{, \theta}=\left\{\frac{c d M_{, \theta}}{\left(a r^{4(1-m)}+c M^{2}\right)}-\frac{2 c^{2} d M_{, \theta} M^{2}}{\left(a r^{4(1-m)}+c M^{2}\right)^{2}}+\frac{d M_{, \theta}}{M^{2}}\right\} r^{2(1-m)}+o\left(r^{2(1-m)}\right) .
\end{gathered}
$$

Operating in the same way, the field equations (2.10) transform into

$$
\begin{gathered}
2 a f_{1}\left[v_{1, \theta \theta}+m^{2} v_{1}\right] r^{(m-2)}+o\left(r^{(m-2)}\right)=-\left[r \psi_{, r} v_{2, \theta} f_{2}-m \psi_{, \theta} v_{2} f_{2}\right] r^{(m-2)} \\
+\rho_{0}\left[v ^ { 2 } \left(m(m-1) v_{1} f_{1} \cos ^{2} \theta-2 m v_{1, \theta} f_{1} \sin \theta \cos \theta+v_{1, \theta \theta} f_{1} \sin ^{2} \theta+2 v_{1, \theta} f_{1} \sin \theta \cos \theta\right.\right. \\
\left.\left.+m v_{1} f_{1} \sin ^{2} \theta\right) r^{(m-2)}-2 v\left(m v_{1} f_{1, t} \cos \theta-v_{1, \theta} f_{1, t} \sin \theta\right) r^{(m-1)}+\varphi_{1, t t}^{0}+v_{1} f_{1, t t} r^{m}\right], \\
2 a f_{2}\left[v_{2, \theta \theta}+m^{2} v_{2}\right] r^{(m-2)}+o\left(r^{(m-2)}\right)=-\left[-r \psi_{, r} v_{1, \theta} f_{1}+m \psi_{, \theta} v_{1} f_{1}\right] r^{(m-2)} \\
+\rho_{0}\left[v ^ { 2 } \left(m(m-1) v_{2} f_{2} \cos ^{2} \theta-2 m v_{2, \theta} f_{2} \sin \theta \cos \theta+v_{2, \theta \theta} f_{2} \sin ^{2} \theta+2 v_{2, \theta} f_{2} \sin \theta \cos \theta\right.\right. \\
\left.\left.+m v_{2} f_{2} \sin ^{2} \theta\right) r^{(m-2)}-2 v\left(m v_{2} f_{2, t} \cos \theta-v_{2, \theta} f_{2, t} \sin \theta\right) r^{(m-1)}+\varphi_{2, t t}^{0}+v_{2} f_{2, t t} r^{m}\right],
\end{gathered}
$$

and the boundary conditions $(2.15)$ become

$$
\begin{aligned}
& {\left[2 a v_{1, \theta}( \pm \pi ; v) f_{1}(t ; v)-m \psi(r, \pm \pi, t) v_{2}( \pm \pi ; v) f_{2}(t ; v)\right] r^{(m-1)}+o\left(r^{(m-1)}\right)=0} \\
& {\left[2 a v_{2, \theta}( \pm \pi ; v) f_{2}(t ; v)+m \psi(r, \pm \pi, t) v_{1}( \pm \pi ; v) f_{1}(t ; v)\right] r^{(m-1)}+o\left(r^{(m-1)}\right)=0}
\end{aligned}
$$

with

$$
\psi(r, \pm \pi, t)=\left\{\frac{c d M( \pm \pi, t)}{a r^{4(1-m)}+c M^{2}( \pm \pi, t)}-\frac{d}{M( \pm \pi, t)}\right\} r^{2(1-m)}+o\left(r^{2(1-m)}\right) .
$$

In the spirit of the asymptotic analysis developed in [7], we divide the field equations (3.3) by $r^{(m-2)}$ and the boundary conditions (3.4) by $r^{(m-1)}$ and proceed to the limit as $r \rightarrow 0$. Since $r \psi_{, r}, \psi_{, \theta}$ and $\psi$ behave like $O\left(r^{2(1-m)}\right)$ as $r \rightarrow 0$ and $m \in(0,1)$, one readily arrives at the following eigenvalue problem:

$$
\begin{gathered}
\left(1-\tilde{v}^{2} \sin ^{2} \theta\right) v_{\alpha, \theta \theta}-\left[\tilde{v}^{2}(1-m) \sin 2 \theta\right] v_{\alpha, \theta}+\left[m^{2}-\tilde{v}^{2} m\left((m-2) \cos ^{2} \theta+1\right)\right] v_{\alpha}=0, \\
v_{\alpha, \theta}( \pm \pi ; \tilde{v})=0,
\end{gathered}
$$

where $\tilde{v}^{2}=\rho_{0} v^{2} /(2 a)$.

Two facts emerge from (3.5): The asymptotic field equations and the related boundary conditions are uncoupled with respect to the angular functions $v_{\alpha}(\theta ; \tilde{v})$; the temporal functions $f_{\alpha}(t ; \tilde{v})$ do not enter into the asymptotic analysis. 
The smallest positive eigenvalue and the associated eigenfunctions admitted by the set of equations (3.5) are [17]

$$
m=\frac{1}{2} \quad \text { and } \quad v_{\alpha}(\theta ; \tilde{v})=\widehat{A}_{\alpha} \operatorname{sgn}(\theta)\left[\frac{\left[1-\tilde{v}^{2} \sin ^{2} \theta\right]^{1 / 2}-\cos \theta}{2}\right]^{1 / 2},
$$

where $\operatorname{sgn}(\theta)=1$ for $0<\theta \leq \pi$ and $\operatorname{sgn}(\theta)=-1$ for $-\pi \leq \theta<0$, and $\widehat{A}_{\alpha}$ denote two arbitrary real constants. For $\hat{v} \leq 1$, the existence of real roots from (3.6) is guaranteed.

The first step of the asymptotic analysis therefore gives the following solution:

$$
\begin{array}{r}
\varphi_{\alpha}(r, \theta, t)=\varphi_{\alpha}^{0}(t)+r^{1 / 2} \widehat{A}_{\alpha} \operatorname{sgn}(\theta)\left[\frac{\left[1-\tilde{v}^{2} \sin ^{2} \theta\right]^{1 / 2}-\cos \theta}{2}\right]^{1 / 2} f_{\alpha}(t ; \tilde{v}) \\
+o\left(r^{1 / 2}\right) \text { as } r \rightarrow 0,
\end{array}
$$

that, like the equivalent static problem [7], implies $M(\theta, t)=0$, providing an inadequate estimate of the determinant of the deformation gradient

$$
\delta=i \lambda \cong o\left(r^{-1}\right) \quad \text { as } \quad r \rightarrow 0 .
$$

It is hence necessary to refine $(3.1)$ by seeking at least a two-term approximation

$$
\begin{aligned}
\varphi_{\alpha}(r, \theta, t)=\varphi_{\alpha}^{0}(t)+ & r^{1 / 2} v_{\alpha}(\theta ; \tilde{v}) f_{\alpha}(t ; \tilde{v})+r^{m^{\prime}} w_{\alpha}(\theta ; \tilde{v}) g_{\alpha}(t ; \tilde{v}) \\
& +o\left(r^{m^{\prime}}\right) \quad \text { as } \quad r \rightarrow 0,
\end{aligned}
$$

where $m^{\prime}>m(=1 / 2), w_{\alpha}(\theta ; \tilde{v})$ and $g_{\alpha}(t ; \tilde{v})$ are as yet undetermined. As in the foregoing computations, $w_{\alpha}(\theta ; \tilde{v})$ and $g_{\alpha}(t ; \tilde{v})$ are twice continuously differentiable real-valued functions that fail to vanish identically on $[-\pi, \pi]$ and on $\left[t_{0}, t_{f}\right)$, respectively. By using the two-term approximation (3.7) of the asymptotic motion field, the above procedure can be repeated by a parallel argument, yielding

$$
\begin{aligned}
& i(r, \theta, t)=Q(\theta, t) r^{\left(m^{\prime}-3 / 2\right)}+M^{\prime}(\theta, t) r^{2\left(m^{\prime}-1\right)}+o\left(r^{2\left(m^{\prime}-1\right)}\right), \\
& \lambda^{2}(r, \theta, t) \\
& =\frac{d}{2\left(a+c Q^{2}(\theta, t) r^{\left(2 m^{\prime}-3\right)}+2 c Q(\theta, t) M^{\prime}(\theta, t) r^{\left(3 m^{\prime}-7 / 2\right)}+c M^{2}(\theta, t) r^{4\left(m^{\prime}-1\right)}\right)} \\
& +o\left(r^{4\left(1-m^{\prime}\right)}\right),
\end{aligned}
$$

where

$$
\begin{gathered}
M^{\prime}(\theta, t)=m^{\prime} g_{1}(t ; \tilde{v}) g_{2}(t ; \tilde{v})\left[w_{1}(\theta ; \tilde{v}) w_{2, \theta}(\theta ; \tilde{v})-w_{1, \theta}(\theta ; \tilde{v}) w_{2}(\theta ; \tilde{v})\right] \\
Q(\theta, t)=f_{1}(t ; \tilde{v}) g_{2}(t ; \tilde{v})\left[\frac{1}{2} v_{1}(\theta ; \tilde{v}) w_{2, \theta}(\theta ; \tilde{v})-m^{\prime} v_{1, \theta}(\theta ; \tilde{v}) w_{2}(\theta ; \tilde{v})\right] \\
+f_{2}(t ; \tilde{v}) g_{1}(t ; \tilde{v})\left[m^{\prime} v_{2, \theta}(\theta ; \tilde{v}) w_{1}(\theta ; \tilde{v})-\frac{1}{2} v_{2}(\theta ; \tilde{v}) w_{1, \theta}(\theta ; \tilde{v})\right] \\
r \psi_{, r}=2 c r i_{, r} \lambda^{2}+4 c r i \lambda \lambda_{, r}+\frac{d i_{, r}}{i^{2}}=O\left(r^{\left(3 / 2-m^{\prime}\right)}\right) \\
\psi_{, \theta}=2 c i_{, \theta} \lambda^{2}+4 c i \lambda \lambda_{, \theta}+\frac{d i_{, \theta}}{i^{2}}=O\left(r^{\left(3 / 2-m^{\prime}\right)}\right) .
\end{gathered}
$$


Bearing in mind the results of the first asymptotic iteration, the field equations $(2.10)$ and the boundary conditions (2.15) provide

$$
\begin{gathered}
2 a g_{1}\left[w_{1, \theta \theta}+m^{\prime 2} w_{1}\right] r^{\left(m^{\prime}-2\right)}+o\left(r^{\left(m^{\prime}-2\right)}\right)=-\left[r \psi_{, r} w_{2, \theta} g_{2}-m^{\prime} \psi_{, \theta} w_{2} g_{2}\right] r^{\left(m^{\prime}-2\right)} \\
+\rho_{0}\left[\tilde { v } ^ { 2 } \left(m^{\prime}\left(m^{\prime}-1\right) w_{1} g_{1} \cos ^{2} \theta-2 m^{\prime} w_{1, \theta} g_{1} \sin \theta \cos \theta+w_{1, \theta \theta} g_{1} \sin ^{2} \theta+2 w_{1, \theta} g_{1} \sin \theta \cos \theta\right.\right. \\
\left.\left.+m^{\prime} w_{1} g_{1} \sin ^{2} \theta\right) r^{\left(m^{\prime}-2\right)}-2 \tilde{v}\left(m^{\prime} w_{1} g_{1, t} \cos \theta-w_{1, \theta} g_{1, t} \sin \theta\right) r^{\left(m^{\prime}-1\right)}+w_{1} g_{1, t t} r^{m^{\prime}}\right], \\
2 a g_{2}\left[w_{2, \theta \theta}+m^{\prime 2} w_{2}\right] r^{\left(m^{\prime}-2\right)}+o\left(r^{\left(m^{\prime}-2\right)}\right)=-\left[-r \psi_{, r} w_{1, \theta} g_{1}+m^{\prime} \psi_{, \theta} w_{1} g_{1}\right] r^{\left(m^{\prime}-2\right)} \\
+\rho_{0}\left[\tilde { v } ^ { 2 } \left(m^{\prime}\left(m^{\prime}-1\right) w_{2} g_{2} \cos ^{2} \theta-2 m^{\prime} w_{2, \theta} g_{2} \sin \theta \cos \theta+w_{2, \theta \theta} g_{2} \sin ^{2} \theta+2 w_{2, \theta} g_{2} \sin \theta \cos \theta\right.\right. \\
\left.\left.+m^{\prime} w_{2} g_{2} \sin ^{2} \theta\right) r^{\left(m^{\prime}-2\right)}-2 \tilde{v}\left(m^{\prime} w_{2} g_{2, t} \cos \theta-w_{2, \theta} g_{2, t} \sin \theta\right) r^{\left(m^{\prime}-1\right)}+w_{2} g_{2, t t} r^{m^{\prime}}\right],
\end{gathered}
$$

and

$$
\begin{aligned}
& {\left[2 a w_{1, \theta}( \pm \pi ; \tilde{v}) g_{1}(t ; \tilde{v})-m^{\prime} \psi(r, \pm \pi, t) w_{2}( \pm \pi ; \tilde{v}) g_{2}(t ; \tilde{v})\right] r^{\left(m^{\prime}-1\right)}+o\left(r^{\left(m^{\prime}-1\right)}\right)=0} \\
& {\left[2 a w_{2, \theta}( \pm \pi ; \tilde{v}) g_{2}(t ; \tilde{v})+m^{\prime} \psi(r, \pm \pi, t) w_{1}( \pm \pi ; \tilde{v}) g_{1}(t ; \tilde{v})\right] r^{\left(m^{\prime}-1\right)}+o\left(r^{\left(m^{\prime}-1\right)}\right)=0}
\end{aligned}
$$

with

$$
\psi(r, \pm \pi, t)=O\left(r^{\left(3 / 2-m^{\prime}\right)}\right) .
$$

We divide Eqs. (3.9) by $r^{\left(m^{\prime}-2\right)}$ and Eqs. (3.10) by $r^{\left(m^{\prime}-1\right)}$ and proceed to the limit as $r \rightarrow 0$. Under the temporary assumption that $m^{\prime}<3 / 2$, the set of equations (3.9) and (3.10) is asymptotically balanced if

$$
\begin{gathered}
\left(1-\tilde{v}^{2} \sin ^{2} \theta\right) w_{\alpha, \theta \theta}-\left[\tilde{v}^{2}\left(1-m^{\prime}\right) \sin 2 \theta\right] w_{\alpha, \theta}+\left[m^{\prime 2}-\tilde{v}^{2} m^{\prime}\left(\left(m^{\prime}-2\right) \cos ^{2} \theta+1\right)\right] w_{\alpha}=0 \\
w_{\alpha, \theta}( \pm \pi ; \tilde{v})=0 .
\end{gathered}
$$

Thus, at the second iteration, one obtains an asymptotic eigenvalue problem which has the same form of the first eigenvalue problem (3.5). In particular, the nonlinear terms again fail to enter the asymptotic analysis explicitly. The smallest eigenvalue $m^{\prime}$ that satisfies the inequality $m^{\prime}>1 / 2$, and the associated eigenfunctions supplied by Eqs. (3.11) are [17]

$$
m^{\prime}=1 \quad \text { and } \quad w_{\alpha}(\theta)=\widehat{B}_{\alpha} \cos \theta
$$

where $\widehat{B}_{\alpha}$ are two arbitrary constants. For $m^{\prime}=1\left(\right.$ and $\left.\left(1-\tilde{v}^{2} \sin ^{2} \theta\right) \neq 0\right)$, the influence of the parameter $\tilde{v}$ disappears.

Finally, with (3.12), the two-term approximation (3.7) of the singular asymptotic motion field around the moving crack-tip assumes the following form:

$$
\begin{aligned}
\varphi_{\alpha}(r, \theta, t)=\varphi_{\alpha}^{0}(t) & +r^{1 / 2} \widehat{A}_{\alpha} \operatorname{sgn}(\theta)\left[\frac{\left[1-\tilde{v}^{2} \sin ^{2} \theta\right]^{1 / 2}-\cos \theta}{2}\right]^{1 / 2} f_{\alpha}(t ; \tilde{v}) \\
& +r \widehat{B}_{\alpha} \cos \theta g_{\alpha}(t ; \tilde{v})+o(r) \text { as } r \rightarrow 0 .
\end{aligned}
$$


Such an asymptotic two-term solution implies

$$
\begin{gathered}
M^{\prime}(\theta, t)=0, \quad i(r, \theta, t)=Q(\theta, t) r^{-1 / 2}+o\left(r^{-1 / 2}\right), \\
\lambda^{2}(r, \theta, t)=\frac{d}{2\left(a r+c Q^{2}(\theta, t)\right)} r+o(r) .
\end{gathered}
$$

From the last two equations we can compute the determinant of the deformation gradient at the moving crack-tip

$$
\delta=\left(\frac{d}{2 c}\right)^{1 / 2}>0,
$$

showing how the asymptotic motion (3.13), for each $t \in \mathcal{I}$, is now an orientationpreserving mapping.

To add higher-order terms to the asymptotic solution (3.13) further iterations can be carried out; these terms, with eigenvalues greater than $m^{\prime}=1$, are however inessential to describe the singular motion field.

Eliminating the time-dependence and setting

$$
\tilde{v}=0, \quad f_{\alpha}(t ; \tilde{v})=g_{\alpha}(t ; \tilde{v})=1,
$$

one immediately obtains from (3.13) the solution of the corresponding static problem [7]:

$$
\varphi_{\alpha}(r, \theta)=\varphi_{\alpha}^{0}+r^{1 / 2} \widehat{A}_{\alpha} \sin \frac{\theta}{2}+r \widehat{B}_{\alpha} \cos \theta+o(r) \quad \text { as } r \rightarrow 0 .
$$

Obviously, the asymptotic solution of the local formulation necessarily contains some unknown terms. Specifically, in the expression (3.13) of the asymptotic motion field, the functions $\varphi_{\alpha}^{0}(t), f_{\alpha}(t ; \tilde{v})$ and $g_{\alpha}(t ; \tilde{v})$, together with the constants $\widehat{A}_{\alpha}$ and $\widehat{B}_{\alpha}$, are undetermined and may be found only after solving the global dynamic crack propagation problem.

\section{Generalization of Stephenson's result and the crack profile during its} propagation. In this section, Stephenson's result is extended to the crack propagation problem treated in this paper. So that, given the solution (3.13), we can retain that the asymptotic motion field at the moving-tip is obtained by applying a rigid rotation $\mathbf{Q}(t)$ to the canonical field $\varphi^{*}(r, \theta, t)$ and adding a rigid translation $\varphi^{0}(t)$, that is,

$$
\boldsymbol{\varphi}(r, \theta, t)=\varphi^{0}(t)+\mathbf{Q}(t) \varphi^{*}(r, \theta, t) .
$$

where $\mathbf{Q}(t)$ has the following components: ${ }^{7}$

$$
[\mathbf{Q}(t)]=\left[\begin{array}{cc}
\frac{\hat{A}_{2} f_{2}(t ; \tilde{v})}{\hat{A}(t)} & \frac{\hat{A}_{1} f_{1}(t ; \tilde{v})}{\hat{A}(t)} \\
-\frac{\hat{A}_{1} f_{1}(t ; \tilde{v})}{\hat{A}(t)} & \frac{\hat{A}_{2} f_{2}(t ; \tilde{v})}{\hat{A}(t)}
\end{array}\right]
$$

with $\widehat{A}(t)=\left(\widehat{A}_{1}^{2} f_{1}^{2}(t ; \tilde{v})+\widehat{A}_{2}^{2} f_{2}^{2}(t ; \tilde{v})\right)^{1 / 2}$. Inverting $(4.1)$, one has

$$
\varphi^{*}(r, \theta, t)=\mathbf{Q}(t)^{\mathrm{T}}\left[\boldsymbol{\varphi}(r, \theta, t)-\varphi^{0}(t)\right]
$$

\footnotetext{
${ }^{7} \mathrm{By}$ virtue of its physical meaning $\widehat{A}(t) \neq 0$, so that $\mathbf{Q}(t)$ preserves the regularity conditions of the functions $f_{\alpha}(t ; \tilde{v})$.
} 
and using (4.2), one readily arrives at the following very special components:

$$
\begin{gathered}
\varphi_{1}^{*}(r, \theta, t)=B_{1}(t ; \tilde{v}) w(\theta) r+o(r), \\
\varphi_{2}^{*}(r, \theta, t)=A_{2}(t ; \tilde{v}) v(\theta ; \tilde{v}) r^{1 / 2}+B_{2}(t ; \tilde{v}) w(\theta) r+o(r),
\end{gathered}
$$

where

$$
\begin{aligned}
B_{1}(t ; \tilde{v}) & =\left[\widehat{A}_{2} \widehat{B}_{1} f_{2}(t ; \tilde{v}) g_{1}(t ; \tilde{v})-\widehat{A}_{1} \widehat{B}_{2} f_{1}(t ; \tilde{v}) g_{2}(t ; \tilde{v})\right] / \widehat{A}(t), \\
A_{2}(t ; \tilde{v}) & =\left[\widehat{A}_{1}^{2} f_{1}^{2}(t ; \tilde{v})+\widehat{A}_{2}^{2} f_{2}^{2}(t ; \tilde{v})\right] / \widehat{A}(t), \\
B_{2}(t ; \tilde{v}) & =\left[\widehat{A}_{1} \widehat{B}_{1} f_{1}(t ; \tilde{v}) g_{1}(t ; \tilde{v})+\widehat{A}_{2} \widehat{B}_{2} f_{2}(t ; \tilde{v}) g_{2}(t ; \tilde{v})\right] / \widehat{A}(t), \\
w(\theta) & =\cos \theta \\
v(\theta ; \tilde{v}) & =\operatorname{sgn}(\theta)\left[\frac{\left[1-\hat{v}^{2} \sin ^{2} \theta\right]^{1 / 2}-\cos \theta}{2}\right]^{1 / 2} .
\end{aligned}
$$

If only the dominant order terms are taken into account, the canonical field (4.3) represents the special motion field in which the crack-faces open symmetrically, satisfying the symmetry properties (1.2).

The function $A_{2}(t ; \tilde{v})$ in $(4.3)_{2}$ plays the same role of the Mode I dynamic stressintensity factor in classic linear elastic fracture mechanics. On the other hand, an analogous function of the Mode II (or shearing mode) does not exist, in that our problem does not admit antisymmetric solutions.

Considering only the dominant order terms in (4.3), the profile of crack surfaces during the propagation is described by the following two parabolic arcs:

$$
\begin{aligned}
\varphi_{2}^{*}(r, \pi, t) & =\left(-\frac{\varphi_{1}^{*}(r, \pi, t)}{B_{1}(t ; \tilde{v})}\right)^{1 / 2} A_{2}(t ; \tilde{v}), \\
\varphi_{2}^{*}(r,-\pi, t) & =-\left(-\frac{\varphi_{1}^{*}(r, \pi, t)}{B_{1}(t ; \tilde{v})}\right)^{1 / 2} A_{2}(t ; \tilde{v}),
\end{aligned}
$$

with $\varphi_{1}^{*}(r, \pi, t)<0, B_{1}(t ; \tilde{v})>0$ and $A_{2}(t ; \tilde{v})>0$, as $r \rightarrow 0$. The two curves join up at the moving-tip with the same tangent, which is perpendicular to the crack-axis. So that, at least in proximity of the crack-tip, the crack-faces are found to open smoothly, without forming cusps.

5. Asymptotic Piola-Kirchhoff and Cauchy stress fields and energy flux. From (2.13), (2.12) and (4.3), the polar and cartesian components of the asymptotic Piola-Kirchhoff stress field are readily obtainable:

$$
\begin{aligned}
& T_{\mathbb{R}_{r r}}^{*}=2 a\left[\frac{1}{2} r^{-1 / 2} A_{2} v \sin \theta+\left(B_{1} \cos \theta+B_{2} \sin \theta\right) \cos \theta\right]+o(1), \\
& T_{\mathbb{R}_{\theta r}}^{*}=2 a\left[\frac{1}{2} r^{-1 / 2} A_{2} v \cos \theta-\left(B_{1} \sin \theta-B_{2} \cos \theta\right) \cos \theta\right]+o(1), \\
& T_{\mathbb{R}_{r \theta}^{*}}^{*}=2 a\left[r^{-1 / 2} A_{2} v_{, \theta} \sin \theta-\left(B_{1} \cos \theta+B_{2} \sin \theta\right) \sin \theta\right]+o(1), \\
& T_{\mathbb{R}_{\theta \theta}}^{*}=2 a\left[r^{-1 / 2} A_{2} v_{, \theta} \cos \theta+\left(B_{1} \sin \theta-B_{2} \cos \theta\right) \sin \theta\right]+o(1),
\end{aligned}
$$


and

$$
\begin{aligned}
& T_{\mathbb{R}_{11}}^{*}=2 a B_{1}+o(1), \\
& T_{\mathbb{R}_{12}}^{*}=o(1), \\
& T_{\mathbb{R}_{21}}^{*}=2 a\left[r^{-1 / 2} A_{2}\left(\frac{1}{2} v \cos \theta-v_{, \theta} \sin \theta\right)+B_{2}\right]+o(1), \\
& T_{\mathbb{R}_{22}}^{*}=2 a\left[r^{-1 / 2} A_{2}\left(\frac{1}{2} v \sin \theta+v_{, \theta} \cos \theta\right)\right]+o(1) .
\end{aligned}
$$

Note that the components $T_{\mathbb{R}_{r \theta}}^{*}$ and $T_{\mathbb{R}_{\theta \theta}}^{*}$ vanish on the crack surfaces as required by the boundary conditions $(2.15)$. From $(5.2)_{1}$ the physical meaning of the function $B_{1}(t ; \tilde{v})$ is also evident.

The cartesian components of the plane deformation gradient, in terms of polar variables, result in

$$
\begin{gathered}
F_{11}^{*}=\varphi_{1, r}^{*} \cos \theta-\varphi_{1, \theta}^{*} \frac{\sin \theta}{r}=B_{1}+o(1), \\
F_{12}^{*}=\varphi_{1, r}^{*} \sin \theta+\varphi_{1, \theta}^{*} \frac{\cos \theta}{r}=o(1), \\
F_{21}^{*}=\varphi_{2, r}^{*} \cos \theta-\varphi_{2, \theta}^{*} \frac{\sin \theta}{r}=A_{2} r^{-1 / 2}\left(\frac{1}{2} v \cos \theta-v_{, \theta} \sin \theta\right)+B_{2}+o(1), \\
F_{22}^{*}=\varphi_{2, r}^{*} \sin \theta+\varphi_{2, \theta}^{*} \frac{\cos \theta}{r}=A_{2} r^{-1 / 2}\left(\frac{1}{2} v \sin \theta+v_{, \theta} \cos \theta\right)+o(1) .
\end{gathered}
$$

With (5.2), (5.3) and using the inverse Piola transform, that is, $\mathbf{T}=\mathbf{T}_{\mathbb{R}}(\mathrm{Cof} \mathbf{F})^{-1}$, the components of the Cauchy stress tensor can be computed:

$$
\begin{gathered}
\delta T_{11}^{*}=2 a B_{1}^{2}+o(1), \\
\delta T_{12}^{*}=\delta T_{21}^{*}=2 a\left[A_{2} B_{1} r^{-1 / 2}\left(\frac{1}{2} v \cos \theta-v_{, \theta} \sin \theta\right)+B_{1} B_{2}\right]+o(1), \\
\delta T_{22}^{*}=2 a\left[A_{2}^{2} r^{-1}\left(\frac{1}{4} v^{2}+v_{, \theta}^{2}\right)+2 A_{2} B_{2} r^{-1 / 2}\left(\frac{1}{2} v \cos \theta-v_{, \theta} \sin \theta\right)+B_{2}^{2}\right]+o(1),
\end{gathered}
$$

where $\delta$ is specified by (3.15).

To investigate the degree of stress singularity in the current configuration we introduce the following spatial radius, evaluated along the line $\theta=0$ :

$$
\rho(r, 0, t)=\left[\varphi_{1}^{*}(r, 0, t)^{2}+\varphi_{2}^{*}(r, 0, t)^{2}\right]^{1 / 2} \cong B(t) r
$$

with $B(t)=\left[B_{1}^{2}(t)+B_{2}^{2}(t)\right]^{1 / 2}$, that substituted into $(5.4)_{3}$ yields

$$
\delta T_{22}^{*} \cong \frac{1}{2} a \frac{A_{2}^{2}}{B}\left(\rho^{-1}\right) .
$$

It follows that, like the corresponding nonlinear static crack problem [7], the most singular component of the Cauchy stress tensor has the asymptotic order $r^{-1}$. 
The mechanical energy removed from the (entire) body during crack propagation may be interpreted in terms of energy flux into the tip of the crack: ${ }^{8}$

$$
\mathbf{j}=\lim _{\left|\Gamma_{t}\right| \rightarrow 0} \int_{\Gamma_{t}}\left[\left(\mathcal{W}+\frac{1}{2} \rho_{0} \dot{\varphi} \cdot \dot{\varphi}\right) \mathbf{I}-\mathbf{F}^{\mathrm{T}} \mathbf{T}_{\mathbb{R}}\right] \mathbf{n}_{\Gamma} d l,
$$

where $\Gamma_{t}$ is a curve surrounding the moving tip $x_{\text {tip }}(t)$ and $\mathbf{n}_{\Gamma}$ is the outward unit normal vector on $\Gamma_{t}$. Expressing the vector of the energy flux (5.7) in terms of the asymptotic canonical field $\varphi^{*}$, one obtains

$$
\mathbf{j}=\lim _{\left|\Gamma_{t}\right| \rightarrow 0} \int_{\Gamma_{t}}\left[\left(\mathcal{W}\left(\mathbf{F}^{*}\right)+\frac{1}{2} \rho_{0}\left(\varphi^{0}+\mathbf{Q} \varphi^{*}\right)^{\bullet} \cdot\left(\varphi^{0}+\mathbf{Q} \varphi^{*}\right)^{\bullet}\right) \mathbf{I}-\mathbf{F}^{* \mathrm{~T}} \mathbf{T}_{\mathbb{R}}^{*}\right] \mathbf{n}_{\Gamma} d l
$$

in that

$$
\mathbf{F}=\mathbf{Q F}^{*}, \quad \mathbf{T}_{\mathbb{R}}=\mathbf{Q} \mathbf{T}_{\mathbb{R}}^{*}, \quad \text { and } \quad \delta=\delta^{*} .
$$

Since the integral (5.8) is path-independent, it may be conveniently computed over a small circle with $d l=r d \theta$, proceeding then to the limit as $r \rightarrow 0$. In this way, only the most singular terms of order $r^{-1}$ help to evaluate the integral (5.8). Substituting (4.3) into (5.8), one arrives, after some computations, at the following components of the j-vector:

$$
\begin{aligned}
& j_{1}=a A_{2}^{2} \int_{-\pi}^{\pi}\left(-\frac{1}{4} v^{2} \cos \theta+v_{, \theta}^{2} \cos \theta+v v_{, \theta} \sin \theta\right) d \theta \\
& j_{2}=a A_{2}^{2} \int_{-\pi}^{\pi}\left(-\frac{1}{4} v^{2} \sin \theta+v_{, \theta}^{2} \sin \theta-v v_{, \theta} \cos \theta\right) d \theta .
\end{aligned}
$$

The integrand of $j_{1}$ is an even function that, given its form, does not seem to be computable in closed-form; however, its numerical evaluation does not present any difficulty. In particular, for $\tilde{v}=0, j_{1}=a A_{2}^{2} \frac{\pi}{2}$ and then decreases as $\tilde{v}$ increases. On the other hand, the integrand of $j_{2}$ is an odd function, so that we obtain, as expected (by virtue of the straight path followed by the moving-tip in the reference configuration) and consistently with the canonical motion field (4.3), $j_{2}=0$, for any value of $\tilde{v}$.

\section{REFERENCES}

[1] R. A. Stephenson, The equilibrium field near the tip of a crack for finite plane strain of incompressible elastic materials, J. Elasticity 12,65-99 (1982)

[2] K. Ch. Le, On the singular elastostatic field induced by a crack in a Hadamard material, Quart. J. Mech. Appl. Math. 45, 101-117 (1992)

[3] K. Ch. Le and H. Stumpf, The singular elastostatic field due to a crack in rubberlike materials, J. Elasticity 32, 183-222 (1993)

[4] P. H. Geubelle and W. G. Knauss, Finite strains at the tip of a crack in a sheet of hyperelastic material: I. Homogeneous case, J. Elasticity 35, 61-98 (1994)

[5] P. H. Geubelle and W. G. Knauss, Finite strains at the tip of a crack in a sheet of hyperelastic material: II. Special bimaterial cases, J. Elasticity 35, 99-138 (1994)

[6] P. H. Geubelle and W. G. Knauss, Finite strains at the tip of a crack in a sheet of hyperelastic material: III. General bimaterial case, J. Elasticity 35, 139-174 (1994)

[7] A. M. Tarantino, Thin hyperelastic sheets of compressible material: field equations, Airy stress function and an application in fracture mechanics, J. Elasticity 44, 37-59 (1996)

\footnotetext{
${ }^{8}$ Total energy flux into the propagation crack-tip was investigated by Freund [15] and Gurtin and Yatomi [16].
} 
[8] J. D. Achenbach and N. Nishimura, Effect of inertia on finite near-tip deformation for fast mode-III crack growth, J. Appl. Mech. 52, 281-286 (1985)

[9] J. D. Achenbach and N. Nishimura, Large deformations near a propagating crack tip, Engineering Fracture Mechanics 23, 183-199 (1986)

[10] P. G. Ciarlet, Mathematical Elasticity. Vol. I: Three-Dimensional Elasticity, North-Holland, 1988

[11] P. G. Ciarlet and G. Geymonat, Sur les lois de comportement en élasticité non-linéaire compressible, C. R. Acad. Sci. Paris Sér. II 295, 423-426 (1982)

[12] J. K. Knowles and Eli Sternberg, Large deformations near a tip of an interface-crack between two Neo-Hookean sheets, J. Elasticity 13, 257-293 (1983)

[13] J. E. Adkins, A. E. Green, and G. C. Nicholas, Two-dimensional theory of elasticity for finite deformation, Philos. Trans. Roy. Soc. A 247, 279-306 (1954)

[14] J. E. Adkins and A. E. Green, Plane problems in second-order elasticity theory, Philos. Trans. Roy. Soc. A 239, 557-576 (1957)

[15] L. B. Freund, Energy flux into the tip of an extending crack in an elastic solid, J. Elasticity 2, 341-349 (1972)

[16] M. E. Gurtin and C. Yatomi, On the energy release rate in elastodynamicsl crack propagation, Arch. Rational Mech. Anal. 74, 231-247 (1980)

[17] A. M. Tarantino, On the finite motions generated by a mode I propagating crack, J. Elasticity 57, 85-103 (1999) 